\title{
Early post-stress decrease in cardiac performance by impedance cardiography and its relationship to the severity and extent of ischemia by myocardial perfusion imaging
}

Ronen Goldkorn ${ }^{1,2^{*}}$ (D) Alexey Naimushin ${ }^{1}$, Eli Rozen ${ }^{1}$ and Dov Freimark ${ }^{1,2}$

\begin{abstract}
Background: While single photon emission computed tomography (SPECT) myocardial perfusion imaging (MPI) is a well-established noninvasive procedure for the evaluation of patients with coronary artery disease (CAD), it is unable to detect the presence of, or underestimates the extent of CAD in certain patients. We aimed to show that a bio-impedance device can detect early post-stress changes in several hemodynamic parameters, thereby serving as a potential marker for the presence of significant ischemia.

Methods: Prospectively enrolled patients, referred to our Medical Center for clinically-indicated MPI, underwent testing using a Non-Invasive Cardiac System (NICaS) before and immediately after exercise. The differences between rest and stress hemodynamic parameters were compared with the severity and extent of myocardial ischemia by MPI. The study included 198 patients; mean age was 62 years, 26\% were women, 54\% had hypertension, and 29\% diabetes mellitus. Of them, 188 patients had $\leq 10 \%$, and 10 had $>10 \%$ of myocardial ischemia.

Results: In the first group, there was a significantly greater increase in post-exercise stroke index, stroke work index, cardiac index and cardiac power index (19.2, 29.1, 90.5 and 107\%, respectively) compared with the second group $(-2.7,3.8,43.7$ and $53.5 \%$, respectively), as well as a significantly greater decrease in total peripheral resistance index $(-38.7 \%$ compared with $-16.3 \%)$, with corresponding $p$ values of $0.015,0.017,0.040,0.016$, and $<0.001$, respectively.
\end{abstract}

Conclusions: Our data suggest that immediate post-stress changes in several hemodynamic parameters, detected by the NICaS, can be used as an important adjunct to SPECT MPI for the early detection of myocardial ischemia.

Keywords: Coronary artery disease, Myocardial perfusion imaging

\footnotetext{
* Correspondence: Ronen.Goldkorn@sheba.health.gov.il

${ }^{1}$ Leviev Heart Center, Sheba Medical Center, 52621 Tel Hashomer, Israel

${ }^{2}$ Sackler School of Medicine, Tel Aviv University, Tel Aviv, Israel
}

C The Author(s). 2020 Open Access This article is licensed under a Creative Commons Attribution 4.0 International License, which permits use, sharing, adaptation, distribution and reproduction in any medium or format, as long as you give appropriate credit to the original author(s) and the source, provide a link to the Creative Commons licence, and indicate if changes were made. The images or other third party material in this article are included in the article's Creative Commons licence, unless indicated otherwise in a credit line to the material. If material is not included in the article's Creative Commons licence and your intended use is not permitted by statutory regulation or exceeds the permitted use, you will need to obtain permission directly from the copyright holder. To view a copy of this licence, visit http://creativecommons.org/licenses/by/4.0/ The Creative Commons Public Domain Dedication waiver (http://creativecommons.org/publicdomain/zero/1.0/) applies to the data made available in this article, unless otherwise stated in a credit line to the data. 


\section{Background}

Single photon emission computed tomography (SPECT) myocardial perfusion imaging (MPI) is a well-established noninvasive procedure for the evaluation and risk stratification of patients with coronary artery disease (CAD) [1]. However, it has been recognized that in certain patients SPECT MPI is unable to detect the presence of, or underestimates the extent of CAD. The fact that moderate to severe perfusion defects are noted in less than half of the patients with significant left main disease [2] has stimulated studies to improve the diagnostic accuracy of SPECT MPI. Such studies have analyzed post-stress parameters such as left ventricular ejection fraction $[3,4]$, wall motion abnormalities [5] and transient left ventricular dilation [6] with images acquired on conventional Anger cameras as long as $60 \mathrm{~min}$ after the stress tracer injection. Such delayed assessment may miss early ischemic stunning as a result of its transient nature.

The Non-Invasive Cardiac System (NICaS, NI Medical, Israel) is a whole-body bio-impedance device capable of measuring various hemodynamic parameters [7-11]. We hypothesized that the non-invasive detection of an early post-stress decrease in cardiac performance may serve as a potential marker for the presence of significant or extensive ischemia. We therefore aimed to test for an early post-stress change in several hemodynamic parameters by the NICaS, and its relationship to the severity and extent of myocardial ischemia in patients undergoing exercise stress MPI using a novel cadmium-zinc-telluride SPECT camera.

\section{Methods}

\section{Study population}

We prospectively enrolled 198 patients who were referred to the Nuclear Cardiology Center at the Sheba Medical Center, Tel Hashomer, Israel, for a clinicallyindicated exercise stress MPI study. Exclusion criteria included patients with: unstable angina, decompensated heart failure, systolic blood pressure $>200 \mathrm{mmHg}$ or diastolic blood pressure $>110 \mathrm{mmHg}$, uncontrolled arrhythmias, severe aortic stenosis, acute pulmonary embolism, acute myocarditis or pericarditis, acute aortic dissection, intra- and extra-cardiac shunts, hemodialysis and those aged $<18$ or $>80$ years of age. The study was approved by the hospital's Institutional Review Board and all patients provided written informed consent.

\section{Exercise stress protocol and image acquisition sequence}

Beta blockers and calcium-channel antagonists were terminated at least $24 \mathrm{~h}$ before testing, and nitrates at least $6 \mathrm{~h}$ before testing. During the pre-imaging stress-lab evaluation and procedures, standard 12 leads for ECG monitoring and leads for image gating were applied, and a venous catheter was inserted into an antecubital vein.
The imaging room was equipped with an ECG monitor, emergency cart and oxygen source.

A "stress-first-rest-second" protocol was used, as previously described [12-21]. Briefly, after obtaining baseline heart rate, blood pressure, and a 12 lead ECG, a symptom-limited treadmill-exercise (Bruce protocol) was performed. At peak exercise, an IV bolus of $6-11 \mathrm{mCi}$ 99mTc-sestamibi, according to a body mass index-related dose schedule, was injected. The first imaging was started at least $20 \mathrm{~min}$ after the IV tracer injection. The patient was placed in the supine position of the cadmium-zinctelluride-SPECT camera (Discovery NM 530c, General Electric Healthcare, Israel). The detector was positioned to include the entire heart image, as well as to isolate the heart from extra-cardiac activity. Acquisitions utilized a $20 \%$ energy window centered around the $140 \mathrm{KeV}$ peak of 99mTc-sestamibi, and a16-bin ECG-gating was performed using a $50 \%$ acceptance window. Subsequently, the patient was placed in a prone position and imaging was repeated. After at least $1.5 \mathrm{~h}$, the patient returned to the lab for rest injection and acquisition using $16-31 \mathrm{mCi}$ of 99mTcsestamibi, according to a body mass index-related dose schedule. Images were reoriented into short-axis and vertical and horizontal long-axis slices using standard software (QPS/QGS, Cedars-Sinai Medical Center, Los Angeles, CA, USA). All image contours were reviewed by experienced technologists and nuclear cardiologists on a case-by-case basis and were individually adjusted if necessary [12-21].

\section{Automated quantification of perfusion}

The QPS software computed the total perfusion deficit score by integrating the hypo-perfusion severities below normal limits in polar map coordinates [14]. Normal limit thresholds were defined as 3.0 mean absolute deviations (approximately equivalent to 2.5 standard deviations) for each polar map sample. Ischemic total perfusion deficit was calculated as an absolute difference between stress and rest total perfusion deficit $[15,16$, 18], and was expressed as a percentage.

\section{The non-invasive cardiac system}

The NICaS calculates the stroke volume by measuring impedance cardiography in a tetra-polar mode, derived from electrodes placed on one wrist and the contralateral ankle $[7,8]$. During transmission of an electrical current through the body, resistivity to its conduction (bio-impedance) is measured. The resistivity of blood and plasma is the lowest in the body, 150 and $63 \Omega / \mathrm{cm}$, respectively, while resistivity of cardiac muscle, lungs and fat is 750,1275 and $2500 \Omega / \mathrm{cm}$, respectively. Thus, when an alternating current of $32.5 \mathrm{kHz}, 1.4 \mathrm{~mA}$ is delivered through the two electrodes, it is primarily distributed via the extracellular fluid and the blood, and the 
changes in body resistivity are therefore related to the dynamic changes of the blood and plasma volume. Therefore, the measured bio-impedance and its fluctuations over time are proportional to the stroke volume. Consequently, each systolic increase in the aortic blood volume is associated with a proportional increase in the measurable conductance of the whole body. In addition, a standard three-lead ECG connection is made for measuring the pulse rate. Patient age, gender, weight, height, hematocrit and electrolytes are entered into the NICaS when the monitoring is started and are used for the stroke volume calculation. Cardiac output is calculated by multiplying stroke volume by the heart rate.

Measurements are adjusted to the body surface area to yield stroke index (SI) and cardiac index (CI). Mean arterial pressure (MAP), calculated from standard blood pressure measurements, together with SI and CI allows the calculation of stroke work index (SWI $=\mathrm{MAP} * \mathrm{SI} /$ $\left.7500.8 \mathrm{~J} / \mathrm{m}^{2}\right)$, cardiac power index $\left(\mathrm{CPI}=\mathrm{MAP}{ }^{*} \mathrm{CI} / 451\right.$ $\left.\mathrm{W} / \mathrm{m}^{2}\right)$ and total peripheral index $\left(\mathrm{TPRI}=\mathrm{MAP} / \mathrm{CI}^{*} 80\right.$ Dyn*Sec $/ \mathrm{cm}^{5 *} \mathrm{~m}^{2}$ ).

This simple to operate, non-invasive technique has been validated in several studies as a reliable estimation of resting $\mathrm{CO}$, compared with traditional, invasive techniques in different settings including healthy subjects, patients with heart failure and ischemia [7-11]. Evaluation of impedance was performed both before and immediately after exercise. Given that the patient needs to be motionless during the measurement to avoid motion artifacts, data acquisition was carried out about $1 \mathrm{~min}$ after completion of exercise. The differences between rest and stress hemodynamic parameters were compared with the severity and extent of myocardial ischemia by MPI.

\section{Statistics}

Statistical analyses were performed using SPSS software (version 2b, IBM Corporation). All baseline variables are described as mean \pm SD. Rest, stress and changes of hemodynamic parameters are described as mean and 95\% confidence interval. A one-way ANOVA was used to compare differences for continuous variables. A chisquare test was used to compare differences across subgroups for categorical variables. A 2-tailed $p<0.05$ as a cut-off was considered statistically significant. Receiver operator characteristic (ROC) curve was used to calculate sensitivity and specificity.

\section{Results}

Patient baseline clinical characteristics and medications are presented in Table 1 . Mean age was $62( \pm 9)$ years and $74 \%$ were males. A sizeable percentage of patients had CAD, with a previous myocardial infarction in $22 \%$, previous percutaneous transluminal coronary angioplasty in $18 \%$, and previous coronary artery bypass grafting in $5 \%$. Notably, the characteristics of those patients with myocardial ischemia $\leq 10 \%$ were similar to those with myocardial ischemia $>10 \%$, with no significant differences in any of the baseline parameters. Patient baseline (resting) hemodynamic parameters are presented in

Table 1 Demographics and general characteristics of all patients and of subgroups (myocardial ischemia $\leq 10$ and $>10 \%$ )

\begin{tabular}{|c|c|c|c|c|}
\hline Parameter & $\begin{array}{l}\text { All patients } \\
\text { N-198 }\end{array}$ & $\begin{array}{l}\text { Myocardial ischemia } \\
\leq 10 \%-\text { N } 188\end{array}$ & $>10 \%-\mathrm{N} 10$ & $P$ value \\
\hline Myocardial ischemia (\%) & $2.4 \pm 3.8 \%$ & $1.8 \pm 2.6 \%$ & $14.4 \pm 3.5 \%$ & $>0.001$ \\
\hline Age (year), mean $\pm S D$ & $61.8 \pm 9.4$ & $61.9 \pm 9.4$ & $60.4 \pm 9.3$ & 0.636 \\
\hline Male, n (\%) & $147(74)$ & $137(72)$ & $10(100)$ & 0.166 \\
\hline $\mathrm{BMI}\left(\mathrm{kg} / \mathrm{m}^{2}\right)$, mean $\pm \mathrm{SD}$ & $27.5 \pm 3.6$ & $27.4 \pm 3.5$ & $29.5 \pm 4.8$ & 0.08 \\
\hline Diabetes mellitus, n (\%) & $58(29.3)$ & $54(28.7)$ & $3(30)$ & 0.923 \\
\hline Hypertension, n (\%) & $106(53.5)$ & $98(52.1)$ & $7(70)$ & 0.265 \\
\hline Smoking, n (\%) & $31(15.7)$ & $30(16.0)$ & $1(10)$ & 0.620 \\
\hline Dyslipidemia, n (\%) & $135(68.2)$ & 128 & $6(60)$ & 0.615 \\
\hline $\mathrm{MI}, \mathrm{n}(\%)$ & $44(22.2)$ & $39(20.7)$ & $4(40)$ & 0.149 \\
\hline PTCA, n (\%) & $35(17.7)$ & $31(16.5)$ & $3(30)$ & 0.268 \\
\hline CABG, n (\%) & $10(5.1)$ & $9(4.8)$ & $1(10)$ & 0.894 \\
\hline PVD, n (\%) & $5(2.5)$ & $5(2.7)$ & 0 & 0.605 \\
\hline TIA, n (\%) & $1(0.5)$ & $1(0.5)$ & 0 & 0.819 \\
\hline Beta Blocker, n (\%) & $52(26.0)$ & $50(26.6)$ & $2(20)$ & 0.653 \\
\hline
\end{tabular}

$B M I$ Body mass index, CABG Coronary artery bypass grafting, MI Myocardial infarction, PTCA Percutaneous transluminal coronary angioplasty, PVD Peripheral vascular disease, SD Standard deviation, TIA Transient ischemic attack 
Table 2 Baseline (rest) hemodynamic parameters of all patients and of subgroups (myocardial ischemia $\leq 10$ and $>10 \%$ )

\begin{tabular}{|c|c|c|c|c|}
\hline Parameter & All patients & $\begin{array}{l}\text { Myocardial ischemia } \\
\leq 10 \%\end{array}$ & $>10 \%$ & $P$ value \\
\hline $\mathrm{SBP}(\mathrm{mmHg})$, mean $\pm \mathrm{SD}$ & $157 \pm 21$ & $157 \pm 21$ & $154 \pm 24$ & 0.729 \\
\hline $\mathrm{DBP}(\mathrm{mmHg})$, mean $\pm \mathrm{SD}$ & $85 \pm 10$ & $85 \pm 11$ & $87 \pm 9$ & 0.967 \\
\hline MAP $(\mathrm{mmHg})$, mean $\pm \mathrm{SD}$ & $108 \pm 12$ & $108 \pm 12$ & $109 \pm 13$ & 0.708 \\
\hline $\mathrm{HR}$ (beats/s), mean $\pm \mathrm{SD}$ & $72 \pm 12$ & $73 \pm 12$ & $69 \pm 6$ & 0.398 \\
\hline $\mathrm{SI}\left(\mathrm{ml} / \mathrm{m}^{2}\right)$, mean $\pm \mathrm{SD}$ & $37.2 \pm 6.7$ & 37.0 & $39.4 \pm 6.1$ & 0.271 \\
\hline $\mathrm{SWI}\left(\mathrm{J} / \mathrm{m}^{2}\right)$, mean $\pm \mathrm{SD}$ & $0.50 \pm 0.10$ & 0.49 & 0.54 & 0.156 \\
\hline $\mathrm{Cl}\left(\mathrm{l} / \mathrm{min} / \mathrm{m}^{2}\right)$, mean $\pm \mathrm{SD}$ & $2.70 \pm 0.63$ & 2.69 & 2.77 & 0.798 \\
\hline $\mathrm{CPI}\left(\mathrm{W} / \mathrm{m}^{2}\right)$, mean $\pm \mathrm{SD}$ & $0.60 \pm 0.15$ & 0.59 & 0.63 & 0.506 \\
\hline TPRI $\left(\right.$ Dyn*S $\left./ \mathrm{cm}^{5 *} \mathrm{~m}^{2}\right)$, mean $\pm \mathrm{SD}$ & $3163 \pm 905$ & 3171 & 3012 & 0.591 \\
\hline $\mathrm{GGl}$, mean $\pm \mathrm{SD}$ & $12.9 \pm 3.5$ & 13.0 & $11.3 \pm$ & 0.133 \\
\hline
\end{tabular}

CPI Cardiac power index, CI Cardiac index, DBP Diastolic blood pressure, GGI Granov Goor index, HR Heart rate, SI Stroke index, MAP Mean arterial pressure, SBP Systolic blood pressure, SWI Stroke work index, TPRI Total peripheral resistance index

Table 2. Once again, there were no statistically significant differences between patients with myocardial ischemia $\leq 10 \%$ and those with myocardial ischemia $>10 \%$.

The hemodynamic changes between rest and stress in patients with myocardial ischemia $\leq 10 \%$ and in those with myocardial ischemia $>10 \%$ are presented in Table 3 . In the first group, there was a significantly greater increase in post-exercise SI, SWI, CI and CPI (19.2, 29.1, 90.5 and $107 \%$, respectively) compared with the second group $(-2.7,3.8,43.7$ and $53.5 \%$, respectively), as well as a significantly greater decrease in TPRI $(-38.7 \%)$ compared with the second group $(-16.3 \%)$, with corresponding $p$ values of $0.015,0.017,0.040,0.016$, and $<0.001$, respectively.

Fig. 1 provides a visual presentation of the change in cardiac function in patients with myocardial ischemia $\leq 10 \%$ and in those with myocardial ischemia $>10 \%$. As may be clearly seen, the SI in the first group increased as expected with an associated marked increase in the CI; however, the SI in the second group actually decreased and thus only a mild increase was noted in the $\mathrm{CI}$, mediated only by the increased heart rate.

Fig. 2 demonstrates the ROC curve for myocardial ischemia $>10 \%$ vs. change in the SI (cut off $=0$ ) from rest

Table 3 Hemodynamic changes between rest and stress in patients with myocardial ischemia $\leq 10$ and $>10 \%$. Data are presented as mean (95\% confidence interval)

\begin{tabular}{|c|c|c|c|}
\hline Parameter & $\begin{array}{l}\text { Myocardial Ischemia } \\
\leq 10 \%\end{array}$ & $>10 \%$ & $P$ Value \\
\hline METS & $9.9(9.5,10.2)$ & $11.2(10.3,12.0)$ & 0.104 \\
\hline Time to target HR & 08:38 (08:17, 08:58) & 09:59 (08:56, 11:02) & 0.074 \\
\hline Max. HR achieved & $147(145,149)$ & $143(137,149)$ & 0.256 \\
\hline$\triangle \mathrm{SBP}(\mathrm{mmHg})$ & $12.5 \%(10.8,14.2 \%)$ & $6.9 \%(1.3,12.4 \%)$ & 0.146 \\
\hline$\triangle \mathrm{DBP}(\mathrm{mmHg})$ & $5.2 \%(3.4,7.0 \%)$ & $7.9 \%(1.3,14.6 \%)$ & 0.499 \\
\hline$\triangle \mathrm{MAP}(\mathrm{mmHg})$ & $8.4 \%(7.0,9.7 \%)$ & $7.8 \%(2.9,12.6 \%)$ & 0.764 \\
\hline$\Delta H R(\text { beats } / \mathrm{s})^{a}$ & $60.0 \%(55.4,64.6 \%)$ & $48.7 \%(26.7,70.7 \%)$ & 0.281 \\
\hline$\Delta \mathrm{SI}(\mathrm{ml} / \mathrm{m} 2)$ & $19.2 \%(15.2,23.2 \%)$ & $-2.7 \%(-8.2,2.7 \%)$ & 0.015 \\
\hline$\Delta \mathrm{SWI}(\mathrm{J} / \mathrm{m} 2)$ & $29.1 \%(24.4,33.8 \%)$ & $3.8 \%(-5.6,13.3 \%)$ & 0.017 \\
\hline$\Delta \mathrm{Cl}(\mathrm{l} / \mathrm{min} / \mathrm{m} 2)$ & $90.5 \%(81.8,99.2 \%)$ & $43.7 \%(22.1,65.3 \%)$ & 0.040 \\
\hline$\Delta \mathrm{CPI}(\mathrm{W} / \mathrm{m} 2)$ & $107 \%(97.2,117 \%)$ & $53.5 \%(33.7,73.3 \%)$ & 0.016 \\
\hline$\Delta \mathrm{TPRI}$ & $-38.7 \%(-41.0,-36.4 \%)$ & $-16.3 \%(-30.7,-1.8 \%)$ & $<0.001$ \\
\hline$\Delta \mathrm{GGl}$ & $22.2 \%(16.7,27.6 \%)$ & $3.8 \%(-3.1,10.6 \%)$ & 0.132 \\
\hline
\end{tabular}

${ }^{\mathrm{a}}$ Hemodynamic measurements were performed with an average delay of 03:30 (03:15, 03:50) minutes from end of treadmill stress. HR was recovered to 114 (111, $116)$ and $98(84,113)$ for the myocardial ischemia $\leq 10$ and $>10 \%$ groups, respectively

CI Cardiac index, CPI Cardiac power index, DBP Diastolic blood pressure, GGI Granov Goor index, HR Heart rate, MAP Mean arterial pressure, METS Metabolic equivalents, SBP Systolic blood pressure, SI Stroke index, SWI Stroke work index, TPRI Total peripheral resistance index 


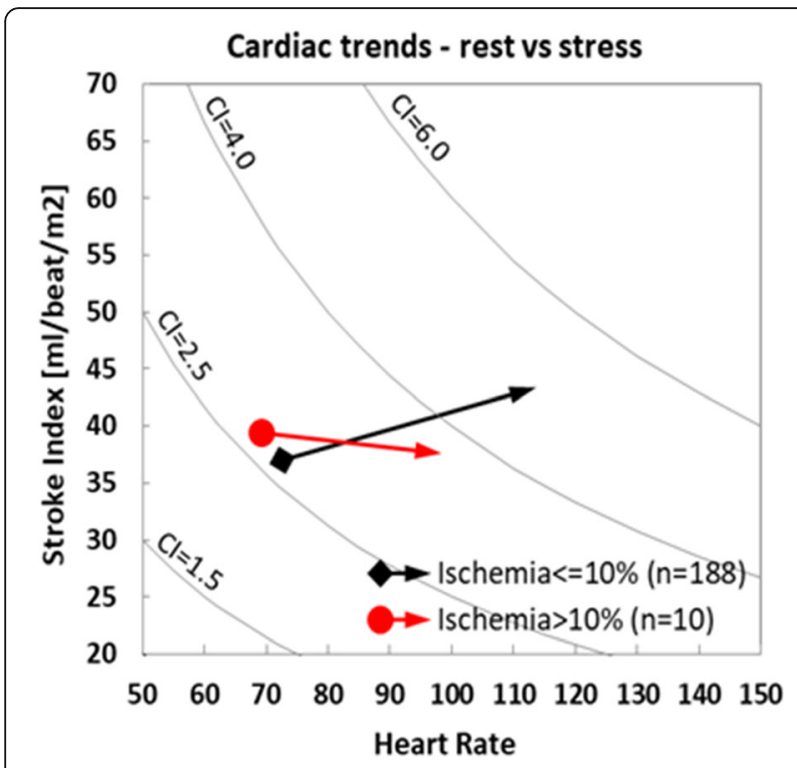

Fig. 1 Stroke index and heart rate trends from rest to stress in patients with myocardial ischemia $\leq 10 \%$ (black arrows) and in those with myocardial ischemia > 10\% (red arrows). Lines of $\mathrm{Cl}$ are also provided. $\mathrm{Cl}=$ Cardiac index

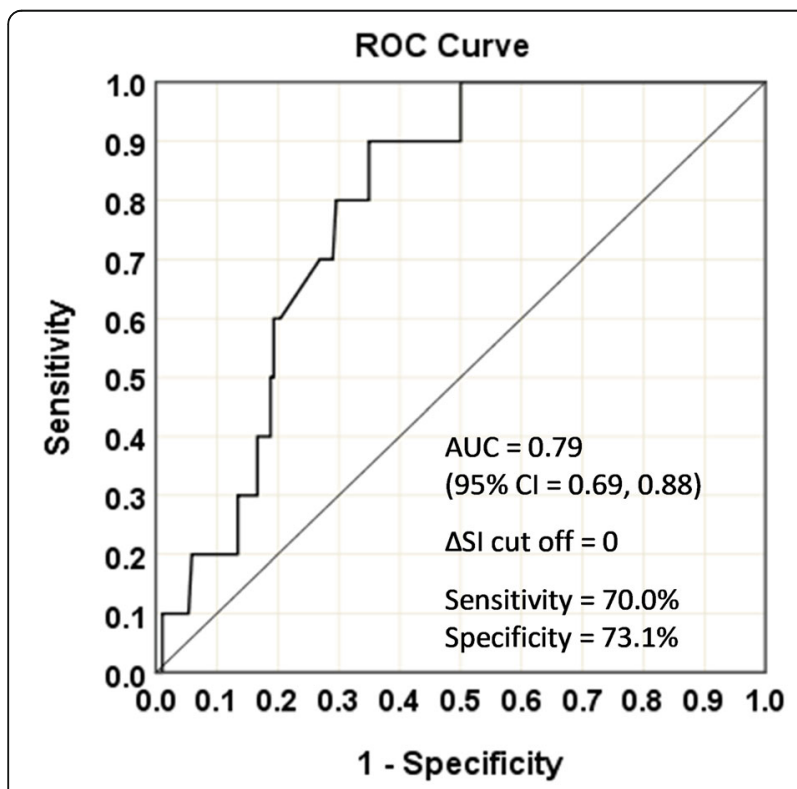

Fig. 2 ROC curve for myocardial ischemia $>10 \%$ vs. change in SI (cut off $=0$ ) from rest to stress. Note the AUC with $95 \% \mathrm{Cl}$, sensitivity, and specificity. $\mathrm{AUC}=$ under the curve; $\mathrm{Cl}=$ confidence interval; $\mathrm{ROC}=$ Receiver operator characteristic; $\mathrm{SI}=$ Stroke index to stress. Noteworthy, are the areas under the curve with 95\% confidence interval, sensitivity, and specificity. As may be seen, a change in SI of $<0$ from rest to stress has $70.0 \%$ sensitivity and $73.1 \%$ specificity for detecting myocardial ischemia of $>10 \%$.

\section{Discussion}

While SPECT MPI is an excellent noninvasive procedure for the detection and risk stratification of patients with CAD, it may be unable to detect the presence of disease in certain patients and may underestimate its severity in others. As a result, several studies have attempted to tackle this issue using various approaches [3-6]. In this prospective study we demonstrate that the non-invasive detection of an early post-stress decrease in cardiac performance may serve as a potential marker for the presence of significant or extensive ischemia.

As already shown in multiple prior studies, the NICaS whole-body bio-impedance device is capable of reliably measuring various hemodynamic parameters [7-11]. In this study we used the NICaS to examine the hemodynamic changes between rest and stress in patients with myocardial ischemia $\leq 10 \%$ and in those with myocardial ischemia $>10 \%$. As seen in Table 3, the patients with ischemia were unable to increase their SWI, CI, and CPI to the same extent as those without significant ischemia, and were unable to decrease TPRI to the same extent as those without significant ischemia.

Most notably, the SI actually decreased in those patients with myocardial ischemia $>10 \%$ (Table 3, Fig. 1). While the SI in patients without significant ischemia increased as expected with an associated marked increase in their CI, it actually decreased in those with ischemia, and therefore only a mild increase was noted in the CI, mediated only by the increased heart rate (Fig. 1). Indeed, any decrease in SI may be used to detect myocardial ischemia $>10 \%$ with $70.0 \%$ sensitivity and $73.1 \%$ specificity (Fig. 2). The novel NICaS technology consistently identifies this pattern and therefore may be an important tool in the detection of ischemia.

The current study certainly has some limitations. Beta-blocker therapy was discontinued at least $24 \mathrm{~h}$ prior to testing and no information was available regarding the use of other medications. In addition, our findings are applicable only to the present study population, comprising patients with an intermediate pretest probability for the presence of CAD without significant comorbidities. Moreover, the small number of patients with myocardial ischemia $>10 \%$ limits certainly impacts the strength of the data. Of note, the reproducibility of the measured hemodynamic parameters may be somewhat limited by the patients' physical fitness and performance on the particular test 
day. Finally, it may also have been useful to have had echocardiographic data to look for valvular comorbidity and to have had additional validation of the stroke volume at rest. We intend to address these limitations in future work as well as to obtain correlation with true anatomy by coronary angiography.

\section{Conclusions}

The results of the present study suggest that the immediate post-stress changes in several hemodynamic parameters as detected by the NICaS can be used as an important adjunct to the diagnostic approach for the early detection of myocardial ischemia. These findings can be used to improve risk assessment prior to a decision regarding the need to proceed with more complex and costly imaging modalities for the detection of myocardial ischemia. Moreover, future research should explore the potential use of the NICaS generated parameters as prognostic markers in the development and evolution of CAD.

\section{Abbreviations}

SPECT: Single photon emission computed tomography; MPI: Myocardial perfusion imaging; CAD: Coronary artery disease; NICaS: Non-invasive cardiac system; SI: Stroke index; Cl: Cardiac index; MAP: Mean arterial pressure; CPI: Cardiac power index; TPRI: Total peripheral resistance index; SWI: Stroke work index; ROC: Receiver operator characteristic

\section{Acknowledgements \\ Poster presentation: \\ Goldkorn, R., Naimushin, A., Rozen, E., and D. Freimark. Early Post-Stress De- crease in Cardiac Performance by Impedance Cardiography and its Relation to the Severity and Extent of Ischemia by Myocardial Perfusion Imaging. European Society of Cardiology International Conference on Nuclear Cardi- ology and Cardiac CT, Lisbon, Portugal 2019. https://academic.oup.com/ehjci- maging/article/20/Supplement_3/jez149.003/5512782}

\section{Authors' contributions}

$R G$, made substantial contributions to the conception and design of the work, the acquisition, analysis, and interpretation of data, and drafted the work. AN, made substantial contributions to the conception and design of the work, the acquisition, analysis, and interpretation of data. ER, made substantial contributions to the the acquisition, analysis, and interpretation of data. DF, made substantial contributions to the conception and design of the work, the acquisition, analysis, and interpretation of data. All authors read and approved the final manuscript.

\section{Funding}

This research received no grant from any funding agency in the public, commercial or not-for-profit sectors.

\section{Availability of data and materials}

All data generated or analyzed during this study are included in this published article.

\section{Ethics approval and consent to participate}

This study was approved by the Sheba Medical Center Institutional Review Board (reference number 2900-16-SMC) and all patients provided written informed consent.

\section{Consent for publication}

Not applicable.

\section{Competing interests}

The authors declare that they have no competing interests.
Received: 5 April 2020 Accepted: 22 July 2020

Published online: 31 July 2020

\section{References}

1. Holly TA, Abbott BG, Al-Mallah M, Calnon DA, Cohen MC, DiFilippo FP, et al. Single photon-emission computed tomography. J Nucl Cardiol. 2010;5:94173.

2. Berman DS, Kang X, Slomka PJ, Gerlach J, de Yang L, Hayes SW, et al. Underestimation of extent of ischemia by gated SPECT myocardial perfusion imaging in patients with left main coronary artery disease. J Nucl Cardiol. 2007:4:521-8.

3. Lima RS, Watson DD, Goode AR, Lima RS, Watson DD, Goode AR, et al. Incremental value of combined perfusion and function over perfusion alone by gated SPECT myocardial perfusion imaging for detection of severe threevessel coronary artery disease. J Am Coll Cardiol. 2003;1:64-70.

4. Sharir T, Germano G, Kavanagh PB, Lai S, Cohen I, Lewin HC, et al. Incremental prognostic value of post-stress left ventricular ejection fraction and volume by gated myocardial perfusion single photon emission computed tomography. Circulation. 1999;10:1035-42.

5. Sharir T, Bacher-Stier C, Dhar S, Lewin HC, Miranda R, Friedman JD, et al. Identification of severe and extensive coronary artery disease by postexercise regional wall motion abnormalities in Tc-99m sestamibi gated single-photon emission computed tomography. Am J Cardiol. 2000;11: $1171-5$.

6. Abidov A, Bax JJ, Hayes SW, Cohen I, Nishina H, Yoda S, et al. Integration of automatically measured transient ischemic dilation ratio into interpretation of adenosine stress myocardial perfusion SPECT for detection of severe and extensive CAD. J Nucl Med. 2004;12:1999-2007.

7. Cohen AJ, Arnaudov D, Zabeeda D, Schulthesis L, Lashinger J, Schachner A, et al. Non invasive measurement of cardiac output during coronary bypass grafting. Eur J Cardiothorac Surg. 1998;14:64-9.

8. Cotter G, Moshkovitz Y, Kaluski E, Cohen AJ, Miller H, Goor D, et al. Accurate, non-invasive, continuous monitoring of cardiac output by whole body electrical bio-impedance. Chest. 2004;125:1431-40.

9. Paredes OL, Shite J, Shinke T, Watanabe S, Otake H, Matsumoto D, et al. Impedance cardiography for cardiac output estimation - reliability of wristto-ankle electrode configuration. Circ J. 2006;70:1164-8.

10. Leitman M, Sucher E, Kaluski E, Wolf R, Peleg E, Moshkovitz Y, et al. Noninvasive measurement of cardiac output by whole-body bio-impedance during dobutamine stress echocardiography: clinical implications in patients with left ventricular dysfunction and ischaemia. Eur J Heart Fail. 2006:8:136-40.

11. Tanino Y, Shite J, Paredes OL, Shinke T, Ogasawara D, Sawada T, et al. Whole body bioimpedance monitoring for outpatient chronic heart failure follow up. Circ J. 2009;73:1074-9.

12. Imbert L, Poussier S, Franken PR, Songy B, Verger A, Morel O, et al. Compared performance of high-sensitivity cameras dedicated to myocardial perfusion SPECT: a comprehensive analysis of phantom and human images. J Nucl Med. 2012;12:1897-903.

13. Sharir T, Slomka PJ, Hayes SW, DiCarli MF, Ziffer JA, Martin WH, et al. Multicenter trial of high-speed versus conventional single-photon emission computed tomography imaging: quantitative results of myocardial perfusion and left ventricular function. J Am Coll Cardiol. 2010;18:1965-74.

14. Erlandsson K, Kacperski K, van Gramberg D, Hutton BF. Performance evaluation of D-SPECT: a novel SPECT system for nuclear cardiology. Phys Med Biol. 2009;9:2635-49.

15. Iskandrian AE, Bateman TM, Belardinelli L, Blackburn B, Cerqueira MD, Hendel RC, et al. ADVANCE MPI investigators. Adenosine versus regadenoson comparative evaluation in myocardial perfusion imaging: results of the ADVANCE phase 3 multicenter international trials. J NuC Cardiol. 2007:5:645-58

16. Sharir T, Ben-Haim S, Merzon K, Prochorov V, Dickman D, Ben-Haim S, et al. High-speed myocardial perfusion imaging. Initial clinical comparison with conventional dual detector anger camera imaging. J Am Coll Cardiol Img. 2008:1:156-63.

17. Patton J, Berman DS, Slomka P. Recent technological advances in nuclear cardiology. J Nucl Cardiol. 2007;14:433-54.

18. Slomka PJ, Nishina H, Berman DS, Akincioglu C, Abidov A, Friedman JD, et al. Automated quantification of myocardial perfusion SPECT using simplified normal limits. J Nucl Cardiol. 2005;1:66-77. 
19. Xu Y, Kavanagh P, Fish M, Gerlach J, Ramesh A, Lemley M, et al. Automated quality control for segmentation of myocardial perfusion SPECT. J Nucl Med. 2009;9:1418-26.

20. Nishina H, Slomka PJ, Abidov A, Yoda S, Akincioglu C, Kang X, et al. Combined supine and prone quantitative myocardial perfusion SPECT: method development and clinical validation in patients with no known coronary artery disease. J Nucl Med. 2006;1:51-8.

21. Brodov $Y$, Fish M, Rubeaux M, Yuka O, Gransar H, Lemley M, et al. Quantitation of left ventricular ejection fraction reserve from early gated regadenoson stress Tc-99m high efficiency SPECT. J Nucl Cardiol. 2016;23: 1251-61.

\section{Publisher's Note}

Springer Nature remains neutral with regard to jurisdictional claims in published maps and institutional affiliations.

Ready to submit your research? Choose BMC and benefit from:

- fast, convenient online submission

- thorough peer review by experienced researchers in your field

- rapid publication on acceptance

- support for research data, including large and complex data types

- gold Open Access which fosters wider collaboration and increased citations

- maximum visibility for your research: over $100 \mathrm{M}$ website views per year

At BMC, research is always in progress.

Learn more biomedcentral.com/submissions 